\title{
Morphological change during crystallization of thin amorphous solid water films on $\operatorname{Ru}(0001)$
}

\author{
Takahiro Kondo and Hiroyuki S. Kato \\ Surface Chemistry Laboratory, RIKEN (The Institute of Physical and Chemical Research), 2-1-1 Wako, \\ Hirosawa, Saitama 351-0198, Japan \\ Mischa Bonn \\ FOM Institute for Atomic and Molecular Physics, Kruislaan 407, 1098 SJ Amsterdam, The Netherlands \\ Maki Kawai \\ Surface Chemistry Laboratory, RIKEN (The Institute of Physical and Chemical Research), 2-1-1 Wako, \\ Hirosawa, Saitama 351-0198, Japan and Department of Advanced Materials Science, University of Tokyo, \\ Kashiwa, Chiba 277-8561, Japan
}

(Received 26 December 2006; accepted 11 April 2007; published online 11 May 2007)

\begin{abstract}
The isothermal crystallization process of thin amorphous solid water (ASW) films on $\mathrm{Ru}(0001)$ has been investigated in real time by simultaneously employing helium atom scattering, infrared reflection absorption spectroscopy, and isothermal temperature-programmed desorption. The measurements reveal that the crystallization mechanism consists of random nucleation events in the bulk of the ASW films, followed by homogeneous growth. Morphological changes of the solid water film during crystallization expose the water monolayer just above the substrate to the vacuum during the crystallization process. (C) 2007 American Institute of Physics. [DOI: 10.1063/1.2739504]
\end{abstract}

Both low-density amorphous solid water (ASW) and crystalline ice (CI) have been observed on planetary bodies and comets, as well as in the interstellar medium and in protoplanetary disks. ${ }^{1,2}$ These ice surfaces provide the catalytic environment for heterogeneous chemical reactions such as the formation of prebiotic organic molecules in the interstellar medium ${ }^{3}$ and reactions that lead to ozone depletion in the stratosphere. ${ }^{4}$ The crystallization processes of ASW and ice surface morphologies therefore have been extensively investigated as prerequisites for the detailed understanding of chemical reactions on ice surfaces. ${ }^{5-17}$

One well-known method of investigating the kinetics of ASW crystallization and the morphology of CI is to monitor the desorption rate of water at a specific temperature (isothermal temperature-programmed desorption, ITPD) during crystallization. ${ }^{5-11}$ In this method, the conversion from ASW to $\mathrm{CI}$ is determined from the change in the desorption rate of water due to different activation barriers of water desorption from ASW and from CI. ${ }^{8,17}$ Quite recently, however, a different interpretation of the ITPD signal was proposed based on time-of-flight secondary ion mass spectroscopy. ${ }^{18,19}$ In these reports, the change in the desorption rate was attributed to morphological changes in ASW and/or phase transformation of ASW to the liquid/supercooled-liquid phase rather than to ASW crystallization. A simultaneous measurement of both the ITPD signal and the phase state of the water layer (by infrared spectroscopy, for example) would conclusively demonstrate which interpretation is correct.

Another debate concerns the surface morphology of CI, which has been examined through the water desorption rate after completion of the crystallization process by ITPD. ${ }^{5-8}$ Different surface morphologies of CI have been reported, depending on the wetting properties of the substrate and the initial water film thickness. For example, the occurrence of zero-order desorption of water from thin CI films on $\mathrm{Pt}(111)$ has been interpreted as the result of the uniform surface morphology of CI owing to the hydrophilic nature of $\mathrm{Pt}(111){ }^{6-8}$ However, an unexpected morphological change of CI on $\mathrm{Pt}(111)$ was recently reported based on measurements of $\mathrm{Kr}$ desorption from the ice surface: ${ }^{10}$ the final water monolayer, which interacts relatively strongly with the $\operatorname{Pt}(111)$ substrate $^{20,21}$ (hereafter referred to as "first water layer"), becomes exposed to the vacuum during zero-order desorption of water from CI, after crystallization has been completed, ${ }^{10}$ but while many water layers remain on the surface.

In the present work, we verify the original interpretation that the ITPD signal reflects the thermodynamic state of the water layer. Furthermore, we find that morphological changes resulting from the formation of three-dimensional (3D) grains of CI causes exposure of the first water layer to the vacuum during the crystallization process of ASW on $\mathrm{Ru}(0001)$. The isothermal crystallization process of ASW layers of $\mathrm{D}_{2} \mathrm{O}$ on $\mathrm{Ru}(0001)$ has been observed in real time by helium atom scattering (HAS), infrared reflection absorption spectroscopy (IRAS), and ITPD simultaneously. This novel combination of techniques is completely noninvasive, causing no damage to the delicate hydrogen-bonded water network. Changes in the surface and bulk phase state are evident from changes in the ITPD and changes in the vibrational response of the water in the IRAS spectra, respectively. HAS is sensitive to changes in morphology and to crystallinity within the topmost molecular layers. The experimental apparatus used in this work has been described elsewhere. ${ }^{22}$ Briefly, the $\mathrm{Ru}(0001)$ surface was cleaned using standard sputtering, annealing, oxidation, and flashing cycles in an ultrahigh vacuum (UHV) chamber with a base pressure 


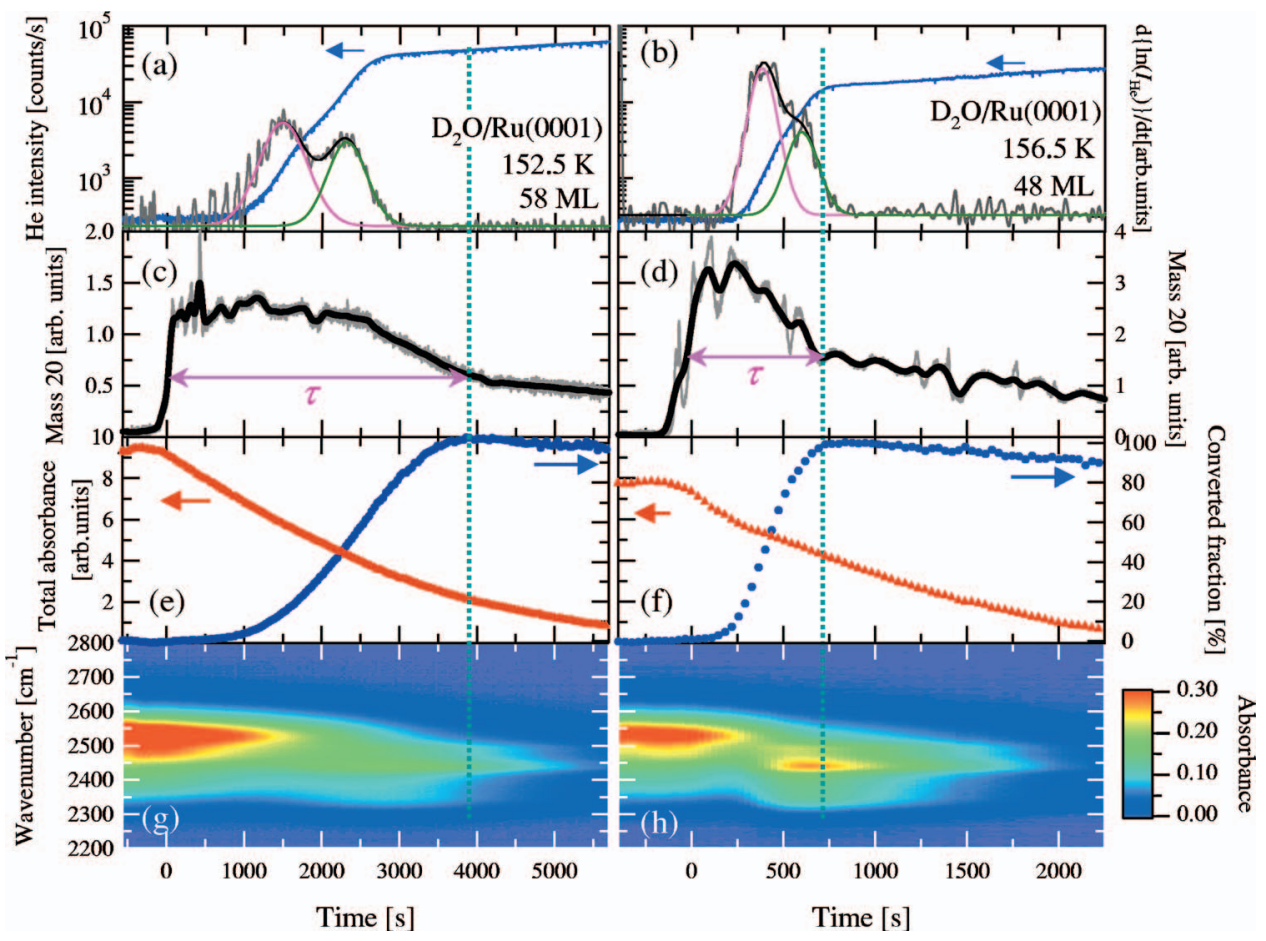

FIG. 1. (Color) Simultaneous acquisitions of HAS, ITPD, and IRAS at $152.5 \mathrm{~K}$ (left) and $156.5 \mathrm{~K}$ (right) as a function of time. During the period up to $t$ $=0 \mathrm{~s}$, the temperature increases from $\sim 90 \mathrm{~K}$ to the designated temperature with a heating rate of $\sim 0.2 \mathrm{~K} / \mathrm{s}$. The temperature is then held at the designated temperature. [(a) and (b)] HAS results [left axis: He beam intensity of the specular reflection, $I_{\mathrm{He}}$, on a log scale. Right axis: $\left[d\left\{\ln \left(I_{\mathrm{He}}\right)\right\} / d t\right.$ (grey curve); black curve represents the fitting result. Red and green curves show the two components of $\left.\left.d\left\{\ln \left(I_{\mathrm{He}}\right)\right\} / d t.\right)\right][(\mathrm{c})$ and $(\mathrm{d})] \operatorname{ITPD}$ results, the desorption rate of $\mathrm{D}_{2} \mathrm{O}$ from $\mathrm{Ru}(0001)$ (grey curve; black curve represents average). [(e) and (f)] IRAS analyzed results (left axis: total weighted absorbance, summation of integrated absorption of the fraction of ASW and that of CI; right axis: converted fraction of ASW to CI phase derived from the linear fit of IRAS result). [(g) and (h)] IRAS results. Each vertical dotted line represents the moment when $\sim 100 \%$ conversion is achieved in (e) and (f), respectively.

of $\sim 1 \times 10^{-10}$ Torr. Thin films of ASW of $\sim 50$ monolayers $^{23}$ (ML) (an order of magnitude larger than the estimated critical nucleus $\operatorname{size}^{8}$ ) were deposited on the surface at $\sim 90 \mathrm{~K}$. Layer thickness is known to affect critically the kinetics of crystallization. ${ }^{5-8}$ For this reason, we report here the kinetics for a fixed initial thickness of $\sim 50 \mathrm{ML}$.

After the deposition of ASW, the layers were heated at a rate of $\sim 0.2 \mathrm{~K} / \mathrm{s}$ to the designated temperature at which ITPD, IRAS, and HAS measurements were made simultaneously. In the HAS measurement, the intensity of specularly reflected $\mathrm{He}$ was measured by a doubly differentially pumped quadrupole mass spectrometer $(\mathrm{QMS}){ }^{22}$ The incident energy and the angle of the helium beam were fixed at $63 \mathrm{meV}$ and $45^{\circ}$, respectively. In the ITPD measurement, desorbed $\mathrm{D}_{2} \mathrm{O}$ molecules were detected by a QMS within the ionization volume in a newly developed home-built small cup (which increases the signal-to-noise ratio) in front of the sample. The substrate temperature was carefully calibrated by temperature-programmed desorption measurements of $\mathrm{D}_{2} \mathrm{O}$ from $\mathrm{Ru}(0001)$ prior to the experiment. ${ }^{24}$ To prevent damage to the water layers by stray electrons from the QMS ionizer, the sample was held at $-160 \mathrm{~V}$ bias during the experiment.

The infrared light from the Fourier transform IR spectrometer (JASCO FT/IR-550) was $p$ polarized by a ZnSe polarizer. It was then focused on the sample surface in the UHV chamber by a concave mirror through the $\mathrm{BaF}$ view port at an $85^{\circ}$ grazing angle of incidence. The specularly reflected IR light from the sample was detected by a mercury-cadmium-telluride detector. All the light paths outside the UHV chamber were purged by pure nitrogen gas to avoid absorption by ambient air, which contains $\mathrm{CO}_{2}$ and $\mathrm{H}_{2} \mathrm{O}$. IRAS spectra were recorded at $4 \mathrm{~cm}^{-1}$ resolution with 20 scan $(40 \mathrm{~s})$ averages. The IR absorbance $A$ is defined as $A=-\ln \left(R / R_{0}\right)$, where $R$ and $R_{0}$ are the reflected intensities with and without the water layers on the substrate, respectively.

We investigated fully deuterated water $\left(\mathrm{D}_{2} \mathrm{O}\right)$ because the IR spectrometer has a better sensitivity in the O-D stretch frequency range than in the range of the $\mathrm{O}-\mathrm{H}$ stretch of $\mathrm{H}_{2} \mathrm{O}$. Whether the $\mathrm{D}_{2} \mathrm{O}$ multilayer is present as ASW or $\mathrm{CI}$ can be determined directly through the O-D stretching vibrational mode $\left(\nu_{\mathrm{OD}}\right)$ of water in the IRAS spectrum as demonstrated previously for $\mathrm{H}_{2} \mathrm{O} .{ }^{16}$ The IR spectra can be reproduced very well by a sum of contributions from amorphous domains (viz, the spectrum at $t=0$ ) and crystalline domains (the spectrum at long times when the IR response remains constant). The "converted fraction" is defined here as the crystalline contribution divided by the weighted sum of the two. ${ }^{16}$

Figure 1 shows simultaneous and in situ observations of HAS, ITPD, and IRAS as a function of time for initially deposited ASW films $\left(\sim 50 \mathrm{ML}_{2} \mathrm{O}\right)$ on $\mathrm{Ru}(0001)$ at 152.5 and $156.5 \mathrm{~K}$. The HAS results depicted by Figs. 1(a) and 1(b) show that the specular HAS intensity increases markedly with time at both temperatures, indicating that signifi- 
cant morphological changes occur at the surface. Simultaneously, a significant drop in the desorption rate occurs, as evidenced by the ITPD signal in Figs. 1(c) and 1(d). When the water desorption rate drops to about half of its initial intensity, the crystallization of ASW is considered complete. ${ }^{5-11}$ The time needed to reach the point indicated as " $~ \tau$ " in Fig. 1 therefore has been used to investigate the crystallization kinetics in detail. ${ }^{6-8}$ As a result of $\mathrm{D}_{2} \mathrm{O}$ desorption, the intensity of the O-D stretch absorption band $\nu_{\mathrm{OD}}$ in the IRAS spectra decreases. For ASW, this absorption band is broad and featureless [Figs. 1(g) and 1(h)] due to strong intramolecular and intermolecular couplings among $\mathrm{D}_{2} \mathrm{O}$ molecules in the hydrogen-bonded network. ${ }^{25,26}$ As the welldocumented IR responses of the crystalline and amorphous phases are different, the fraction of ASW converted into CI can be extracted readily from IRAS spectra. The results are shown in Figs. 1(e) and 1(f). At each temperature, the point at which the converted fraction reaches $\sim 100 \%$ coincides with a change in the desorption rate, as indicated by the dotted line in Figs. 1(c)-1(f). This proves explicitly the validity of using ITPD to monitor crystallization, and excludes another, recently proposed interpretation of ITPD, which attributes the change in the desorption rate in ITPD to morphological change and/or phase transformation to the liquid phase. ${ }^{18,19}$

From Fig. 1, we also can infer the nature of the nucleation of ASW crystallization. If the crystallization is nucleated predominantly at the outermost ASW surface, the drop in ITPD [Figs. 1(c) and 1(d)] should occur well before the point at which $100 \%$ bulk conversion has occurred, as observed by IRAS [Figs. 1(e)-1(h)], because the desorption occurs only from the surface molecules exposed to the vacuum. On the other hand, if nucleation occurs at the RuASW interface, the HAS intensity [Figs. 1(a) and 1(b), which probes the outermost surface] should not change in the initial stages of crystallization. The fact that neither of these phenomena is observed in the results depicted in Fig. 1 indicates a random nucleation event in the bulk of ASW. This nucleation mechanism has been used to explain the crystallization of ASW on most substrates reported to date based on the analysis of the crystallization kinetics. ${ }^{5-15}$ One marked exception is $\mathrm{ASW}$ on $\mathrm{CI} / \mathrm{Pt}(111)$, for which heterogeneous nucleation was attributed to the template effect of the substrate as a two-dimensional nucleation site for the growth of CI. ${ }^{12,13,17}$ In the case of $\mathrm{Ru}(0001)$, it has been anticipated that epitaxial growth of $\mathrm{CI}$ on $\mathrm{Ru}(0001)$ may occur due to the small lattice mismatch between the $\mathrm{Ru}(0001)$ substrate and CI. ${ }^{20,21}$ One therefore might expect a similar template effect on $\mathrm{Ru}(0001)$ as well. Our results shown in Fig. 1, however, clearly exclude this template effect on $\mathrm{Ru}(0001)$.

To confirm our conclusion and to derive more details of the crystallization mechanism, we have measured the temperature dependence of the crystallization mechanism/ process and analyzed the results using theoretical calculations. The conversion rates at $153 \mathrm{~K}$ derived from measured IRAS spectra are shown in Fig. 2(a). According to the classical model of nucleation and growth of isothermal solidstate phase transformation kinetics, the isothermal time dependence of the crystallization mole fraction is given
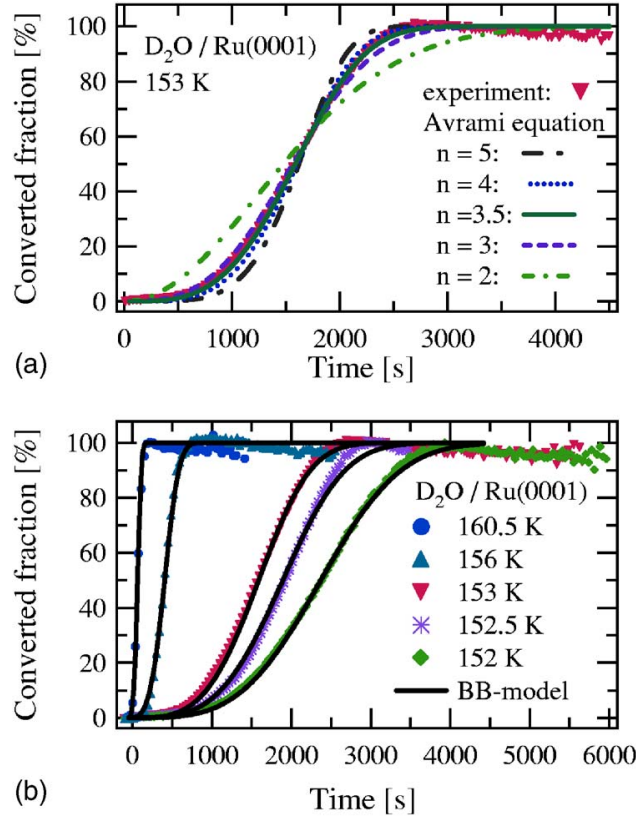

FIG. 2. (Color online) Converted fraction from ASW to CI calculated from the analysis of IRAS spectra. (a) The result at $153 \mathrm{~K}$ is analyzed using the Avrami-type equation (see text), where the equation with $n=3.5$ reproduces our result. (b) Results at various temperatures are shown as a function of isothermal annealing time. Solid curves are the calculated results based on the Backus and Bonn model (BB model) (see text).

by the following Avrami-type equation: ${ }^{27,28} \quad \chi(t)=100$ $\left[1-\exp \left\{-(k t)^{n}\right\}\right]$, where $\chi$ is the converted fraction [\%], $t$ is time, $k$ is a crystallization rate constant, and $n$ is a parameter that depends on the mechanism of the crystallization. The best fit of our results to the above equation is for $n=\sim 3.5$, as shown in Fig. 2(a). When heterogeneous nucleation occurs, $n$ is known to be $\sim 1.4,{ }^{17}$ while $n=\sim 4$ corresponds to a mechanism involving spatially random bulk nucleation with a constant nucleation rate and isotropic 3D growth of the grains at a constant radial rate. ${ }^{27,28}$ Our derived value of $n$ $=\sim 3.5$ is therefore consistent with the conclusion drawn above that crystallization of ASW occurs through random nucleation in the bulk, followed by effectively isotropic 3D growth.

The above conclusion of the crystallization mechanism enables us to analyze our results by a more quantitative theory formulated recently by Backus and Bonn. ${ }^{29}$ The theory includes the following three potentially important effects: (i) the desorption of the material, (ii) the finite nucleation core size, and (iii) the possibility that nucleation occurs at the ASW-substrate interface or the ASW-vacuum interface. This theory agrees closely with our experimental results, as shown by the solid curves in Fig. 2(b), where a nucleation grain diameter of $3 \mathrm{ML}$ is used ${ }^{8}$ and the rates of bulk nucleation and homogeneous growth are adjustable parameters. The derived kinetic parameters of the crystallization are summarized in Table. $\mathrm{I}^{30}$ with the desorption rate inferred from the time variation of the IRAS total absorbance. The excellent agreement with the experimental temperature dependence shown in Fig. 2(b) conclusively demonstrates that the crystallization mechanism consists of random 
TABLE I. The kinetic parameters of bulk nucleation rate $\left[\mathrm{ML}^{3} / \mathrm{s}\right]$ and growth rate $[\mathrm{ML} / \mathrm{s}]$ derived from the fitting analysis (see text). The desorption rate $[\mathrm{ML} / \mathrm{s}]$ used in the calculation is estimated from the time variation of the IRAS total absorbance.

\begin{tabular}{cccc}
\hline \hline $\begin{array}{c}\text { Temperature } \\
(\mathrm{K})\end{array}$ & $\begin{array}{c}\text { Desorption rate } \\
(\mathrm{ML} / \mathrm{s})\end{array}$ & $\begin{array}{c}\text { Growth rate } \\
(\mathrm{ML} / \mathrm{s})\end{array}$ & $\begin{array}{c}\text { Bulk nucleation rate } \\
\left(\mathrm{ML}^{3} / \mathrm{s}\right)\end{array}$ \\
\hline 152 & $1.28 \times 10^{-2}$ & $4.3 \times 10^{-2}$ & $7.20 \times 10^{-9}$ \\
152.4 & $1.41 \times 10^{-2}$ & $4.6 \times 10^{-2}$ & $1.3 \times 10^{-8}$ \\
153.0 & $1.33 \times 10^{-2}$ & $4.8 \times 10^{-2}$ & $2.00 \times 10^{-8}$ \\
155.0 & $2.99 \times 10^{-2}$ & 0.10 & $1.85 \times 10^{-7}$ \\
156.0 & $3.31 \times 10^{-2}$ & 0.11 & $2.70 \times 10^{-7}$ \\
160.6 & $2.37 \times 10^{-2}$ & 0.25 & $4.00 \times 10^{-6}$ \\
\hline \hline
\end{tabular}

nucleation events in the bulk of the material, followed by effectively homogeneous growth.

The Arrhenius-type plot presented in Fig. 3 is based on the time required for the $100 \%$ conversion from ASW to CI as determined from IRAS analysis. The derived apparent activation energy for the crystallization of bulk ASW $(\sim 50$ ML) is $650 \pm 25 \mathrm{meV}$, which is similar to previous reports based on the ITPD analysis. ${ }^{5,7}$

In addition to details of the mechanism and kinetics of ASW crystallization, we can derive information about the morphological change of the outermost surface during ASW crystallization from the measurements shown in Fig. 1. The initial HAS intensity in Fig. 1, after thin film growth, is limited by background counts; due to the disordered nature of the ASW surface, there is no significant specular intensity. During crystallization, the increase of the HAS intensity over time may be due to two factors: (1) the appearance of CI domains that are sufficiently ordered for efficient He scattering and (2) the exposure of the substrate domains to the vacuum. Indeed, closer inspection of the HAS signal reveals the presence of two distinct contributions to the signal. This is evident most clearly from the time derivative of the signal shown in Figs. 1(a) and 1(b), which shows a double-peaked structure for both temperatures. The first occurs at the same time as the onset of crystallization; the second thus likely represents the exposure of the substrate to the vacuum. ${ }^{31}$ This conclusion is corroborated by measurements of water crystallization on the CO-precovered $\mathrm{Ru}(0001)$ surface, which is the topic of a future publication. Note that the morphological change resulting in the exposure of the substrate

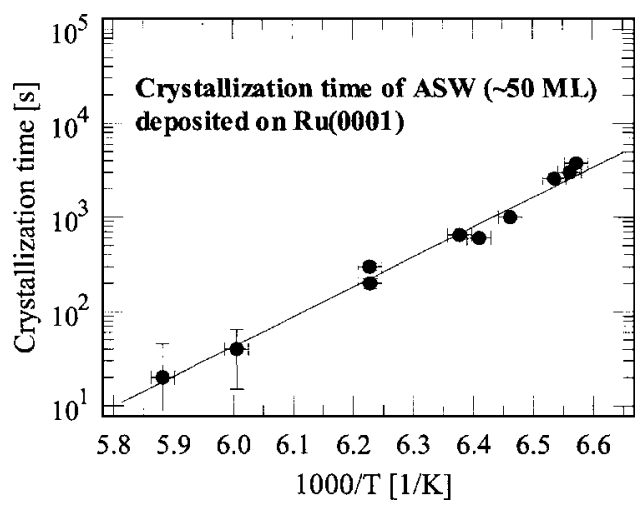

FIG. 3. Arrhenius plot of the ASW ( $\sim 50 \mathrm{ML})$ crystallization time $(100 \%$ conversion time) derived from the IRAS analysis (see text).

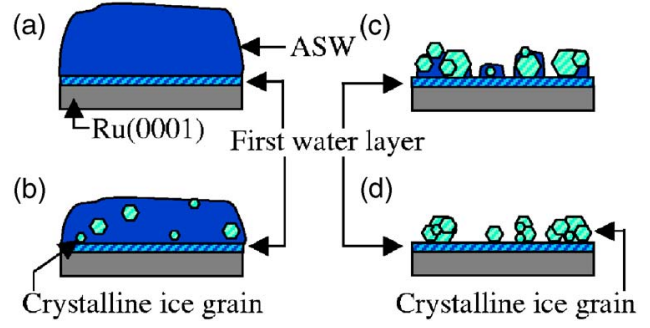

FIG. 4. (Color online) Schematic diagram of the isothermal crystallization process of $\mathrm{ASW}(\sim 50 \mathrm{ML})$ on $\mathrm{Ru}(0001)$ observed by our measurements (see text).

occurs during crystallization, i.e., faster than $\tau$.

Here, the "substrate" exposed to the vacuum is considered the first water layer on $\mathrm{Ru}(0001)$. The structure and the stability of the first water layer on $\mathrm{Ru}(0001)$ have been extensively investigated (see Ref. 32 and references therein). Taking care to avoid ambient electrons impinging onto the surface, only two desorption peaks can be observed in the temperature-programmed desorption of $\mathrm{D}_{2} \mathrm{O}$ from $\mathrm{Ru}(0001)$ (Ref. 32) as we previously reported. ${ }^{24,33}$ Peaks appearing in the TPD spectrum at $\sim 160$ and $\sim 180 \mathrm{~K}$ have been assigned to multilayer water and first water layer desorption, respectively. The first water layer interacts relatively strongly with the substrate. Since the experiments shown in Figs. 1 and 2 are conducted well below the desorption temperature of the first water layer, that layer must be left on the surface under our experimental conditions.

The ASW crystallization process on $\mathrm{Ru}(0001)$ is schematically summarized in Fig. 4. Randomly nucleated CI grains grow at a specific rate depending on temperature [Fig. 4(b)]. Simultaneously, molecular rearrangement among the water molecules results in morphological change. This rearrangement is presumably caused by the different binding energies of water molecules to $\mathrm{CI}$ and $\mathrm{ASW}$, and therefore must be induced by the nucleation of CI grains [Fig. 4(c)]. The large-scale morphological change of the ice surface that occurs during the crystallization process gives rise to the exposure of the first water layer to the vacuum [Figs. 4(c) and 4(d)].

In summary, we have presented the simultaneous and in situ observations of the isothermal crystallization process of ASW layers on $\mathrm{Ru}(0001)$ by HAS, IRAS, and ITPD. The original interpretation of the ITPD measurement clearly has been verified: the ITPD signal provides a good measure for the thermodynamic state of the water layer. Our measurements also reveal that the crystallization mechanism consists of random nucleation events in the bulk of the ASW films, followed by homogeneous growth. The apparent activation energy of the crystallization of bulk ASW film ( $\sim 50 \mathrm{ML})$ is found to be $650 \pm 25 \mathrm{meV}$. Morphological changes of the ASW film have been found to expose the first water layer to the vacuum during the crystallization process.

One of the authors (T. K.) gratefully acknowledges financial support from the SPR System in RIKEN (20032005). This research was also partially supported by the RIKEN Research Program "Nanoscale Science and Technology Research" and by the Ministry of Education, Culture, 
Sports, Science and Technology (MEXT) through a "Grantin-Aid for Young Scientists (B) 17760034, 2005-2007."

${ }^{1}$ E. F. van Dishoeck, Annu. Rev. Astron. Astrophys. 42, 119 (2004).

${ }^{2}$ P. Ehrenfreund, H. J. Fraser, J. Blum, J. H. E. Cartwright, J. M. GarciaRuiz, E. Hadamcik, A. C. Levasseur-Regourd, S. Price, F. Prodi, and A. Sarkissian, Planet. Space Sci. 51, 473 (2003).

${ }^{3}$ P. Ehrenfreund, W. Irvine, L. Becker et al., Rep. Prog. Phys. 65, 1427 (2002).

${ }^{4}$ J. P. D. Abbatt, Chem. Rev. (Washington, D.C.) 103, 4783 (2003).

${ }^{5}$ R. S. Smith, C. Huang, E. K. L. Wong, and B. D. Kay, Surf. Sci. 367, L13 (1996).

${ }^{6}$ P. Löfgren, P. Ahlström, D. V. Chakarov, J. Lausmaa, and B. Kasemo, Surf. Sci. 367, L19 (1996).

${ }^{7}$ P. Löfgren, P. Ahlström, J. Lausma, B. Kasemo, and D. Chakarov, Langmuir 19, 265 (2003).

${ }^{8}$ P. Ahlström, P. Löfgren, J. Lausma, B. Kasemo, and D. Chakarov, Phys. Chem. Chem. Phys. 6, 1890 (2004).

${ }^{9}$ G. Zimbitas, S. Haq, and A. Hodgson, J. Chem. Phys. 123, 174701 (2005).

${ }^{10}$ G. A. Kimmel, N. G. Petrik, Z. Dohnálek, and B. D. Kay, Phys. Rev. Lett. 95, 166102 (2005).

${ }^{11}$ A. S. Bolina, A. J. Wolff, and W. A. Brown, J. Phys. Chem. B 109, 16836 (2005).

${ }^{12}$ Z. Dohnálek, R. L. Ciolli, G. A. Kimmel, K. P. Stevenson, R. S. Smith, and B. D. Kay, J. Chem. Phys. 110, 5489 (1999).

${ }^{13}$ Z. Dohnálek, G. A. Kimmel, R. L. Ciolli, K. P. Stevenson, R. S. Smith, and B. D. Kay, J. Chem. Phys. 112, 5932 (2000).

${ }^{14}$ D. J. Safarik, R. J. Meyer, and C. B. Mullins, J. Chem. Phys. 118, 4660 (2003).

${ }^{15}$ D. J. Safarik and C. B. Mullins, J. Chem. Phys. 121, 6003 (2004).

${ }^{16}$ E. H. G. Backus, M. Grecea, A. W. Kleyn, and M. Bonn, Phys. Rev. Lett. 92, 236101 (2004).

${ }^{17}$ R. S. Smith, Z. Dohnálek, G. A. Kimmel, G. Teeter, P. Ayotte, J. L. Daschbach, and B. D. Kay, in Water in Confining Geometries, edited by V. Buch and J. P. Devlin (Springer-Verlag, Berlin, 2003), pp. 337-357.

${ }^{18}$ R. Souda, J. Phys. Chem. B 110, 14787 (2006).

${ }^{19}$ R. Souda, J. Phys. Chem. B 110, 17524 (2006).

${ }^{20}$ P. A. Thiel and T. E. Madey, Surf. Sci. Rep. 7, 211 (1987).
${ }^{21}$ M. A. Henderson, Surf. Sci. Rep. 46, 1 (2002).

${ }^{22}$ T. Kondo, H. S. Kato, T. Yamada, S. Yamamoto, and M. Kawai, Eur. Phys. J. D 38, 129 (2006).

${ }^{23}$ The thickness of the water layer is expressed in monolayers (ML). $1 \mathrm{ML}$ is defined as the amount of water adsorbed on the $\mathrm{Ru}(0001)$ surface that in a thermal desorption experiment gives rise to one desorption feature near $180 \mathrm{~K}$ (Ref. 24); this feature saturates with exposure, after which multilayer desorption occurs near $160 \mathrm{~K}$.

${ }^{24}$ T. Kondo, S. Mae, H. S. Kato, and M. Kawai, Surf. Sci. 600, 3570 (2006).

${ }^{25}$ W. Hagen, A. G. G. M. Tielens, and J. M. Greenberg, Chem. Phys. 56, 367 (1981)

${ }^{26}$ J. P. Devlin, J. Geophys. Res. 106, 33333 (2001).

${ }^{27}$ M. J. Avrami, J. Chem. Phys. 7, 1103 (1939); 8, 212 (1940); 9, 177 (1941).

${ }^{28}$ R. H. Doremus, Rates of Phase Transformations (Academic, New York, 1985).

${ }^{29}$ E. H. G. Backus and M. Bonn, J. Chem. Phys. 121, 1038 (2004).

${ }^{30}$ An Arrhenius plot prepared from the temperature dependence of the bulk nucleation rate and the growth rate, derived from the fitting analysis, yields activation energies of $E_{\text {nucleation }}=1556 \mathrm{meV}$ and $E_{\text {growth }}=438 \mathrm{meV}$. Both values are in reasonable agreement with the values reported in the ASW crystallization on $\mathrm{Pt}(111)$ (Ref. 13) and on $\operatorname{Ir}(111)$ (Ref. 15). Our derived activation energies, however, may be only one of several possible combinations of fitting parameters (Ref. 14). For an exact determination, one of the parameters should be set using one of the experimental or theoretical methods such as the method described by Safarik and Mullins for the analysis of surface crystallization of ASW (Ref. 15).

${ }^{31}$ In the case of Fig. 1(a), the moment when crystallization is complete $(\sim 3900 \mathrm{~s})$ is impossible to determine from the HAS signal, although ITPD and IRAS show significant changes of the surface and bulk phase states, respectively. These changes probably originate from the smaller contribution of the scattering intensity from the CI domain than from the first water layer in the HAS intensity components, though the precise origin is still unclear.

${ }^{32}$ N. S. Faradzhev, K. L. Kostov, P. Feulner, T. E. Madey, and D. Menzel, Surf. Sci. 415, 165 (2005).

${ }^{33}$ M. M. Thiam, T. Kondo, N. Horimoto, H. S. Kato, and M. Kawai, J. Phys. Chem. B 109, 16024 (2005). 